\title{
Is Liver Cirrhosis a Risk Factor for Osteonecrosis of the Femoral Head in Adults? A Population-Based 3-Year Follow-Up Study
}

\author{
Tsung-Hsing Hung ${ }^{1,2}$, Yu-Hsi Hsieh ${ }^{1,2}$, Chih-Chun Tsai ${ }^{3}$, Chih-Wei Tseng ${ }^{1,2}$, \\ Kuo-Chih Tseng ${ }^{1,2}$ and Chen-Chi Tsai ${ }^{2,4}$
}

\begin{abstract}
Background The relationship between osteonecrosis of the femoral head (OFH) and liver cirrhosis is controversial. The aim of this study was to determine whether liver cirrhosis is associated with the occurrence of $\mathrm{OFH}$.

Methods We used the National Health Insurance Database, derived from the Taiwan National Health Insurance program. The study cohort comprised 40,769 adult patients with liver cirrhosis. The comparison cohort consisted of 40,769 randomly selected age- and sex-matched subjects.

Results During the 3-year follow-up period, there were $321(0.8 \%)$ cirrhotic patients with OFH, and 126 $(0.3 \%)$ non-cirrhotic patients with OFH ( $\mathrm{p}<0.001)$. Cox's regression analysis, adjusted by the patients' age, sex, and other confounding factors, showed that the cirrhotic patients had a higher risk for occurrence of $\mathrm{OFH}$ than non-cirrhotic patients during the 3 -year period (hazard ratio $=2.38, \mathrm{p}<0.001$ ). In this 3 -year study, the incidence density of cirrhotic patients hospitalized for OFH was 3 episodes/1,000 person-year.

Conclusion We conclude that cirrhotic patients have a higher risk for occurrence of OFH than non-cirrhotic patients.
\end{abstract}

Key words: osteonecrosis, femoral head, liver cirrhosis

(Intern Med 50: 2563-2568, 2011)

(DOI: 10.2169/internalmedicine.50.5952)

\section{Introduction}

Osteonecrosis of the femoral head (OFH) is a condition characterized by destruction and collapse of the femoral head. In many atraumatic patients, OFH develops insidiously, progresses to collapse of the femoral head, and then causes rapid destruction of the hip joint. There are many reported risk factors, including trauma, alcohol abuse, steroid overuse, venous outflow obstruction, systemic lupus erythematosus, vasculitis, radiation therapy, arterial thrombosis or embolism, sick cell anemia and myeloproliferative disorder (1-12). Sakaguchi's study showed a positive association of liver disease with idiopathic $\mathrm{OFH}$, but the significance is marginal (6). A similar phenomenon was noted in our clinic population; however, it is still unknown whether liver cirrhosis itself, independent of alcohol consumption, is a risk factor for OFH. Because atraumatic OFH is not a common disease, it is difficult to collect sufficient cases to verify this association in a single hospital. In order to overcome this problem, we used a nationwide population-based database to examine the occurrence of $\mathrm{OFH}$ in cirrhotic and noncirrhotic patients during a 3-year follow-up.

\footnotetext{
${ }^{1}$ Division of Gastroenterology, Department of Medicine, Buddhist Dalin Tzu Chi General Hospital, Taiwan, ${ }^{2}$ School of Medicine, Tzu Chi University, Taiwan, ${ }^{3}$ Department of Mathematics, Tamkang University, Taiwan and ${ }^{4}$ Division of Infectious Diseases, Department of Medicine, Buddhist Dalin Tzu Chi General Hospital, Taiwan

Received for publication June 4, 2011; Accepted for publication July 11, 2011

Correspondence to Dr. Chen-Chi Tsai, antibody_1@msn.com
} 


\section{Patients and Methods}

\section{Database}

The data was from the Taiwan National Health Insurance research database. The Taiwan National Health Insurance Bureau, in cooperation with the National Health Research Institute, established and maintained this database. This database was used to identify all hospital discharges in Taiwan. The National Health Insurance program in Taiwan was developed in 1995 for all citizens who live in Taiwan. In 2004, the National Health Insurance covered more than 95\% of Taiwan's population. All researchers who use the National Health Insurance research database are required to be evaluated by the National Health Research Institute. Researchers are required to sign a permit to protect the privacy of patients. The present study analyzing this database had been approved by the National Health Research Institute, and has been assigned application and agreement Number: 99247.

\section{Study sample}

This was a retrospective cohort study. Patients who were discharged with a primary or accessory diagnosis of liver cirrhosis (ICD-9-CM code 571.5, or 571.2) from 1 January 2004 to 31 December 2004 were identified from the database. Because of the natural history of liver cirrhosis, we excluded patients younger than 30 years of age. Because biliary liver cirrhosis (ICD-9-CM code 571.6) is a liver disease of a different mechanism, cases of biliary liver cirrhosis were not included in our study. In addition, patients with incomplete or missing basic data in this database were not included. Totally, 40,769 cirrhotic patients without baseline OFH were enrolled in this cohort study. The comparison group was composed of other patients in the same database. We randomly selected 40,769 sex- and age-matched noncirrhotic patients $(30-44,45-59,60-74$ and $>75)$. Each patient was followed individually for a 3-year period starting at the time of the first hospitalization, and continuing until OFH was identified (ICD-9-CM codes 733.42).

Other risks for $\mathrm{OFH}$ were counted if the condition was noted at the time of the first admission, including alcoholism (ICD-9-CM codes 291, 303, 305.00-305.03, 571.0571.3), diabetes mellitus (DM) (ICD-9-CM code 250), hip joint trauma (femoral neck fracture: ICD-9-CM codes 820 or dislocation of hip joint: ICD-9-CM codes 835), gouty arthritis (ICD-9-CM codes 274), human immunodeficiency virus (HIV) infection (ICD-9-CM code 042), chronic renal failure (CRF) (ICD-9-CM code 585), rheumatoid arthritis (RA) (ICD-9-CM code 714), pancreatitis (ICD-9-CM codes 577.0, 577.1), hyperlipidemia (ICD-9-CM codes 272), solid organ transplantation (ICD-9-CM codes V42.0, V42.1, V42.7), and connective tissue disease (ICD-9-CM codes 710).

\section{Survival time}

To determine the time to $\mathrm{OFH}$, survival time was defined as the time from the first hospitalization to the time of hospitalization for $\mathrm{OFH}$, with patients censored at death, or the end of the study.

\section{Statistical analyses}

The SPSS statistical package (SPSS System for Windows, version 13.0) was used to perform the analyses in this study. Univariate analysis was performed with Chi-square testing for other risk factors of $\mathrm{OFH}$ between cirrhotic and noncirrhotic patients. Kaplan-Meier analysis was used to construct survival plots of time to hospitalized OFH after the first admission. Stepwise regression (likelihood ratio method) was used to model factors associated with time to hospitalized OFH, controlling for age and all covariates. Finally, we present hazard ratios (HR) along with the $95 \%$ confidence intervals (CI) using a significance level of 0.05 for this study.

\section{Results}

Table 1 presents the distribution of demographic characteristics and selected risk factors of $\mathrm{OFH}$ at baseline between cirrhotic and non-cirrhotic patients. All patients were older than 30 years old (mean: 59.3 years; standard deviation: 14.3 years). There were 16,247 (39.9\%) patients with complicated cirrhosis (ascites, episodes of esophageal/gastric variceal bleeding, or hepatic encephalopathy) and 24,522 $(60.1 \%)$ patients with non-complicated cirrhosis. Of 81,538 patients, 447 patients $(0.5 \%)$ experienced $\mathrm{OFH}$ during the 3 year follow-up period: 321 cirrhotic patients $(0.8 \%)$ and 126 non-cirrhotic patients $(0.3 \%)(\mathrm{p}<0.001)$. Of 321 the cirrhotic patients with $\mathrm{OFH}, 153(47.7 \%)$ belonged to complicated cirrhosis and $168(52.3 \%)$ non-complicated cirrhosis. The incidence density of hospitalization for $\mathrm{OFH}$ in cirrhotic patients was 3.0 episodes/1000 person-years, and was 1.1 episodes/1000 person-years in non-cirrhotic patients. The mean follow-up period for cirrhotic patients was 953 days, and 1,036 days for non-cirrhotic patients $(\mathrm{p}<0.001)$. The median time between the first admission and the onset of $\mathrm{OFH}$ was 366 days for cirrhotic patients, compared with the 503 days for non-cirrhotic patients.

In the Cox regression analysis, the results are provided in Table 2. After adjusting for gender, age, and other confounding factors, the hazard ratio of $\mathrm{OFH}$ during 3-year follow-up period was 2.38 (95\% $\mathrm{CI}=1.89-2.99, \mathrm{p}<0.001)$. CRF, RA, HIV infection, pancreatitis, solid organ transplantation, and hyperlipidemia were not independent risk factors in this study. Furthermore, patients with hip trauma (hazard ratio, 8.87; 95\% CI, 4.84-16.24; $\mathrm{p}<0.001$ ), connective tissue disease (hazard ratio, 10.58; 95\% CI, 4.32-25.92; $<<0.001$ ), alcoholism (hazard ratio, 1.93; 95\% CI, 1.54-2.42; $<<0.001$ ), diabetes (hazard ratio, 0.60; 95\% CI, 0.43-0.82; $\mathrm{p}=0.001$ ), and gout (hazard ratio, 1.91; 95\% CI, 1.25-2.90; $\mathrm{p}=0.003$ ) 
Table 1. Demographic Characteristics and Comorbid Medical Disorders for Patients with Cirrhosis and Comparison Patients $(n=81538)$

\begin{tabular}{|c|c|c|c|}
\hline & $\begin{array}{l}\text { Cirrhotic patients } \\
\quad(n=40769)\end{array}$ & $\begin{array}{c}\text { Comparison patients } \\
(\mathrm{n}=40769)\end{array}$ & $\mathrm{p}$ value \\
\hline Male, no. (\%) & $28978(71.1)$ & $28978(71.1)$ & 1.000 \\
\hline \multicolumn{4}{|l|}{ Age, yr } \\
\hline 30-44, no. $(\%)$ & $7051(17.3)$ & $7051(17.3)$ & 1.000 \\
\hline $45-59$, no. $(\%)$ & $13057(32.0)$ & $13057(32.0)$ & 1.000 \\
\hline $60-74$, no. $(\%)$ & $14149(34.7)$ & $14149(34.7)$ & 1.000 \\
\hline$>75$, no. $(\%)$ & $6512(16.0)$ & $6512(16.0)$ & 1.000 \\
\hline Hip trauma, no. (\%) & $138(0.3)$ & $453(1.1)$ & $<0.001^{\mathrm{a}}$ \\
\hline Diabetes, no. $(\%)$ & $8027(19.7)$ & $7178(17.6)$ & $<0.001^{\mathrm{a}}$ \\
\hline CRF, no. $(\%)$ & $1250(3.1)$ & $1150(2.8)$ & $0.038^{\mathrm{a}}$ \\
\hline $\begin{array}{l}\text { Connective tissue disease, no. } \\
(\%)\end{array}$ & $77(0.2)$ & $60(0.1)$ & 0.171 \\
\hline Alcoholism, no. $(\%)$ & $7924(19.4)$ & $673(1.7)$ & $<0.001^{\mathrm{a}}$ \\
\hline RA, no. $(\%)$ & $50(0.1)$ & $150(0.3)$ & $<0.001^{\mathrm{a}}$ \\
\hline Gout, no. (\%) & $800(2.0)$ & $1082(2.7)$ & $<0.001^{\mathrm{a}}$ \\
\hline Pancreatitis, no. (\%) & $1190(2.9)$ & $566(1.4)$ & $<0.001^{\mathrm{a}}$ \\
\hline Hyperlipidemia, no. (\%) & $421(1.0)$ & $1549(3.8)$ & $<0.001^{\mathrm{a}}$ \\
\hline HIV infection, no. (\%) & $15(0.0)$ & $20(0.0)$ & 0.499 \\
\hline SOT, no. $(\%)$ & $129(0.3)$ & $77(0.2)$ & $<0.001^{\mathrm{a}}$ \\
\hline
\end{tabular}

Table 2. Adjusted Hazard Ratios for Osteonecrosis of the Femoral Head during the 3-year Follow-up Starting from the First Hospitalization

\begin{tabular}{lccr}
\hline \multicolumn{4}{c}{ Occurrence of osteonecrosis of the femoral head } \\
\hline Variables & Hazard ratio & $95 \%$ confidence interval & p value \\
\hline Liver cirrhosis & 2.38 & $1.89-2.99$ & $<0.001$ \\
Gender (male) & 1.71 & $1.26-2.33$ & 0.001 \\
Age & 0.95 & $0.95-0.96$ & $<0.001$ \\
Hip trauma & 8.87 & $4.84-16.24$ & $<0.001$ \\
Connective tissue disease & 10.58 & $4.32-25.92$ & $<0.001$ \\
Alcoholism & 1.93 & $1.54-2.42$ & $<0.001$ \\
Gout & 1.91 & $1.25-2.90$ & 0.003 \\
Diabetes & 0.60 & $0.43-0.82$ & 0.001 \\
\hline
\end{tabular}

were independent association factors for OFH. Male sex and younger age were associated with a greater likelihood of OFH. The log-rank test indicated that cirrhotic patients had significantly higher occurrence of OFH within 3-year follow-up than non-cirrhotic patients $(\mathrm{p}<0.001)$. Time to hospitalization for $\mathrm{OFH}$ in cirrhotic and non-cirrhotic patients in the 3-year follow-up is shown in the Fig. 1.

\section{Discussion}

In the present study, the incidence density of OFH in cirrhotic patients is higher than in non-cirrhotic patients. However the occurrence rate is very low. Because this difference is small, a previous study did not demonstrate a statistically significant difference $(13,14)$. The large sample size in the present study provides the statistical power to detect the risk of occurrence of $\mathrm{OFH}$ in cirrhotic patients. Liver transplantation was previously proven to be associated with OFH (15). However, the etiologies of cirrhosis in this study were mainly primary biliary cirrhosis and primary sclerosing cholangitis, which sometimes needed steroid therapy. It is still not clear whether liver cirrhosis itself is associated with OFH. In Taiwan, most cases of cirrhosis were due to hepati- tis $\mathrm{B}$ or $\mathrm{C}$ virus infection, especially hepatitis $\mathrm{B}$ virus $(16,17)$. In our study, $19.4 \%$ cirrhotic patients were attributed to alcohol, and the remaining $80.6 \%$ was attributed to viral hepatitis. This distribution was compatible with previously published data in Taiwan $(16,17)$. In clinical practice, steroid and other immunosuppressive agents were avoided as much as possible in patients with cirrhosis due to viral hepatitis. Because most cases of cirrhosis were due to viral hepatitis in the present study, the effects of alcohol and steroid usage can be diminished.

In the present study, an increased risk of OFH was also noted in patients with joint trauma, male sex, younger age, heavy alcohol use, and gouty arthritis. These findings are compatible with previous studies $(18,19)$; however, questions may be raised with regard to the fact that our confounding factors did not include dosage of steroids, which is considered to be an important factor for OFH $(3,5,13,18-24)$. Through this database, it was impossible for us to collect the data about the dosage and duration of steroid usage. So, we choose diseases treated with longterm steroids as confounding factors, such as solid organ transplantation, connective tissue disease, or rheumatoid arthritis. In addition, steroids were always avoided in patients 


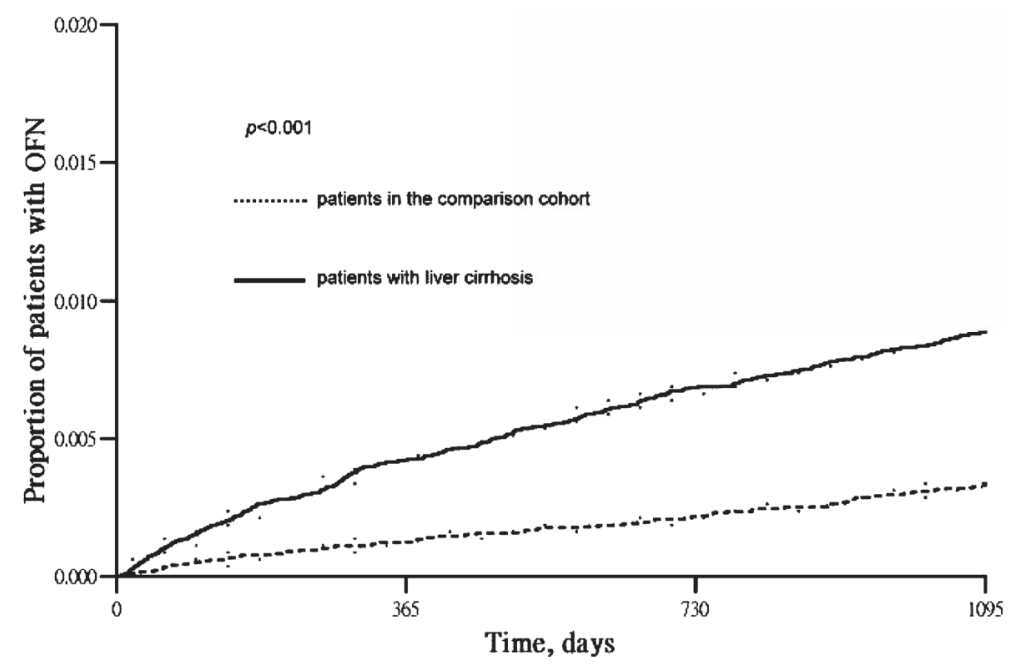

Figure 1. Time to hospitalization for osteonecrosis of the femoral head (OFN) in cirrhotic patients $(n=40769)$ and comparison patients $(n=40769)$ after 3-year follow-up. The proportions of cirrhotic patients and comparison patients who experienced OFN after a 3-year follow-up were $0.8 \%$ and $0.3 \%$, respectively (p value $<0.001$ ).

with viral hepatitis. Although we did not account for steroid use per se in our study, the effect of this confounding factor is negligible.

Despite the fact that many associated factors that have been proposed, the pathogenesis of the disease is not well understood. Impairment of the vascular supply to the femoral neck is considered as an important mechanism for OFH (25). This results in death of the bone marrow and trabecular bone, and eventual bone collapse. A process of reparative reaction is then initiated, but unless the lesion is small or medially located, this repair process is usually ineffective (26). OFN may be caused by other mechanisms other than impairment of vascular supply. Our study showed an interesting phenomenon that DM is negatively correlated with the occurrence of $\mathrm{OFH}$. The same phenomenon was also noted in a previous study (27). DM is a risk factor of atherosclerotic vascular disorder (28). However, DM is not a risk factor for OFN. Similarly, liver cirrhosis associated with impairment of coagulation and thrombocytopenia is proved to be a risk factor for $\mathrm{OFH}$.

Liver cirrhosis itself has been proven to cause a loss of bone mass due to poor cholecalciferol absorption through the intestine, poor activation of vitamin $\mathrm{D}$, and chronic hypogonadism in men (29-32); however, osteoporosis itself appears insufficient to cause OFH. Interleukin-6 (IL-6) is a proinflammatory cytokine. Overexpression of IL-6 can drive chronic joint inflammation and lead to arthritis $(33,34)$. In addition, IL-6 can induce osteoclastogenesis and bone resorption (35-37). Elevation of serum IL-6 is found in alcohol-related cirrhotic patients (38). Similarly, elevation of serum IL-6 is also found in HBV-related or HCV-related cirrhotic patients (39). A study also proved impaired hepatic clearance of IL-6 in cirrhotic patients (40). We consider that the higher osteoclastogenesis induced by higher IL- 6 is the most important reason for atraumatic OFN in cirrhotic pa- tients. This may cause bone resorption and mechanosensory disturbances with subsequent collapse of bone. However, further study will be needed to prove more evidence to support this theory.

In this study, we noted a high ratio of comparison patients with hip trauma. We considered this to be due to the sex- and age-matched study design. We needed to collect many non-cirrhotic inpatients aged $<60$ years. In this age group, trauma was a major reason for hospitalization. That is the reason why there was a high ration of hip trauma in the comparison group. This study has several limitations. First, the database we selected does not include complete information that may be relevant to our study, such as smoking, the dosage and duration of steroid use, or alcohol consumption, any of which can cause $\mathrm{OFH}$; however, we selected related medical comorbidities, such as alcoholism and connective tissue disease to diminish possible bias. In addition, nearly all published etiologies of $\mathrm{OFH}$ were chosen for analysis in our study. Secondly, the true frequency of OFH may be underestimated in this study, because not all patients with OFH require hospital admission; however, hospitalization for OFH would be a marker for a disabling or more severe form of $\mathrm{OFH}$, which may have more important clinical significance. Third, in our study, we can not discern the severity of liver cirrhosis according to ICD-9 coding. Despite these limitations, our study is the most complete nationwide population-based study for identifying the risk of OFH in patients with cirrhosis. In conclusion, our study demonstrates that cirrhotic patients have higher risk for OFH than non-cirrhotic patients. Physicians should be aware of the importance of evaluating hip pain in cirrhotic patients.

The authors state that they have no Conflict of Interest (COI). 


\section{Acknowledgement}

This study is based in part on data from the National Health Insurance Research Database provided by the Bureau of National Health Insurance, Department of Health and managed by National Health Research Institutes (Registered number: 99247). The interpretation and conclusions contained herein do not represent those of the Bureau of National Health Insurance, Department of Health or National Health Research Institutes.

\section{References}

1. Bachiller FG, Caballer AP, Portal LF. Avascular necrosis of the femoral head after femoral neck fracture. Clin Orthop Relat Res 399: 87-109, 2002.

2. Mankin HF. Nontraumatic necrosis of bone (osteonecrosis). N Engl J Med 326: 1473-1479, 1993.

3. Weinstein RS, Nicholas RW, Manolagas SC. Apoptosis of osteocytes in glucocorticoid-induced osteonecrosis of the hip. J Clin Endocrinol Metab 85: 2907-2912, 2000.

4. Assouline-Dayan Y, Chang C, Greenspan A, Shoenfeld Y, Gershwin ME. Pathogenesis and natural history of osteonecrosis. Semin Arthritis Rheum 32: 94-124, 2000.

5. Wang GJ, Cui Q, Balian G. The Nicolas Andry award. The pathogenesis and prevention of steroid-induced osteonecrosis. Clin Orthop Relat Res 370: 295-310, 2000.

6. Hernigou P, Habibi A, Bachir D, Galacteros F. The natural history of asymptomatic osteonecrosis of the femoral head in adults with sickle cell disease. J Bone Joint Surg Am 88: 2565-2572, 2006.

7. Wang Y, Li Y, Mao K, Li J, Cui Q, Wang GJ. Alcohol-induced adipogenesis in bone and marrow: a possible mechanism for osteonecrosis. Clin Orthop Relat Res 410: 213-224, 2003.

8. Abu-Shakra M, Buskila D, Shoenfeld Y. Osteonecrosis in patients with SLE. Clin Rev Allergy Immunol 25: 13-24, 2003.

9. Glueck CJ, Freiberg R, Tracy T, Stroop D, Wang P. Thrombophilia and hypofibrinolysis: pathophysiologies of osteonecrosis. Clin Orthop Relat Res 334: 43-56, 1997.

10. Massin P, Duparc J. Total hip replacement in irradiated hips. A retrospective study of 71 cases. J Bone Joint Surg Br 77: 847-852, 1995.

11. Wang TY, Avlonitis EG, Relkin R. Systemic necrotizing vasculitis causing bone necrosis. Am J Med 84: 1085-1086, 1988.

12. Mont MA, Hungerford DS. Non-traumatic avascular necrosis of the femoral head. J Bone Joint Surg Am 77: 459-474, 1995.

13. Sakaguchi M, Tanaka T, Fukushima W, Kubo T, Hirota Y; Idiopathic ONF. Multicenter Case-Control Study Group. Impact of oral corticosteroid use for idiopathic osteonecrosis of the femoral head: a nationwide multicenter case-control study in Japan. J Orthop Sci 15: 185-191, 2010.

14. Shibata A, Fukuda K, Inoue A, et al. Flushing pattern and idiopathic avascular necrosis of the femoral head. J Epidemiol 6: 37 43, 1996.

15. Guichelaar MM, Schmoll J, Malinchoc M, Hay JE. Fractures and avascular necrosis before and after orthotopic liver transplantation: long-term follow-up and predictive factors. Hepatology 46: 11981207, 2007.

16. Hsu HC, Lin WS, Tsai MJ. Hepatitis-B surface antigen and hepatocellular carcinoma in Taiwan. With special reference to types and localization of HBsAg in the tumor cells. Cancer 52: 18251832, 1983.

17. Tsai JF, Chang WY, Jeng JE, et al. Hepatitis $C$ virus infection as a risk factor for non-alcoholic liver cirrhosis in Taiwan. J Med Virol 41: 296-300, 1933.

18. Fukushima W, Fujioka M, Kubo T, Tamakoshi A, Nagai M, Hirota Y. Nationwide epidemiologic survey of idiopathic osteonecrosis of the femoral head. Clin Orthop Relat Res 468: 2715-2724, 2010.
19. Giacomello A, Zoppini A, Sorgi ML, Riccieri V, Salerno C. Hyperuricemia, gout and idiopathic aseptic necrosis of bone. Adv Exp Med Biol 253A: 211-214, 1989.

20. Koo KH, Kim R, Kim YS, et al. Risk period for developing osteonecrosis of the femoral head in patients on steroid treatment. Clin Rheumatol 21: 299-303, 2002.

21. Horiuchi H, Hashikura Y, Hisa K, et al. Osteonecrosis of the femoral head in Japanese adults after liver transplantation: a preliminary report. J Orthop Sci 9: 119-121, 2004.

22. Allison GT, Bostrom MP, Glesby MJ. Osteonecrosis in HIV disease: epidemiology, etiologies, and clinical management. AIDS 17: 1-9, 2003.

23. Marston SB, Gillingham K, Bailey RF, Cheng EY. Osteonecrosis of the femoral head after solid organ transplantation: a prospective study. J Bone Joint Surg Am 84: 2145-2151, 2002.

24. Chang CC, Greenspan A, Gershwin ME. Osteonecrosis: current perspectives on pathogenesis and treatment. Semin Arthritis Rheum 23: 47-69, 1993.

25. Jones LC, Hungerford DS. Osteonecrosis: etiology, diagnosis, and treatment. Curr Opin Rheumatol 16: 443-449, 2004.

26. Mont MA, Jones LC, Einhorn TA, Hungerford DS, Reddi AH. Osteonecrosis of the femoral head. Potential treatment with growth and differentiation factors. Clin Orthop Relat Res 355: S314-S335, 1998.

27. Abbott KC, Oglesby RJ, Agodoa LY. Hospitalized avascular necrosis after renal transplantation in the United States. Kidney Int 62: 2250-2256, 2002.

28. Yamagishi S, Nakamura K, Takeuchi M, Imaizumi T. Molecular mechanism for accelerated atherosclerosis in diabetes and its potential therapeutic intervention. Int J Clin Pharmacol Res 24: 129134, 2004.

29. Carey EJ, Balan V, Kremers WK, Hay JE. Osteopenia and osteoporosis in patients with end-stage liver disease caused by hepatitis $\mathrm{C}$ and alcoholic liver disease: not just a cholestatic problem. Liver Transpl 9: 1166-1173, 2003.

30. Guichelaar MM, Kendall R, Malinchoc M, Hay JE. Bone mineral density before and after OLT: long-term follow-up and predictive factors. Liver Transpl 12: 1390-1402, 2006.

31. Janes $\mathrm{CH}$, Dickson ER, Okazaki R, Bonde S, McDonagh AF, Riggs BL. Role of hyperbilirubinemia in the impairment of osteoblast proliferation associated with cholestatic jaundice. J Clin Invest 95: 2581-2586, 1995.

32. Guichelaar MM, Malinchoc M, Sibonga JD, Clarke BL, Hay JE. Bone histomorphometric changes after liver transplantation for chronic cholestatic liver disease. J Bone Miner Res 18: 21902199, 2003.

33. McInnes IB, Schett G. Cytokines in the pathogenesis of rheumatoid arthritis. Nat Rev Immunol 7: 429-442, 2007.

34. Smolen JS, Beaulieu A, Rubbert-Roth A, et al. Effect of interleukin-6 receptor inhibition with tocilizumab in patients with rheumatoid arthritis (OPTION study): a double-blind, placebocontrolled, randomised trial. Lancet 371: 987-997, 2008.

35. Wong PK, Quinn JM, Sims NA, van Nieuwenhuijze A, Campbell IK, Wicks IP. Interleukin-6 modulates production of $T$ lymphocyte-derived cytokines in antigen-induced arthritis and drives inflammation-induced osteoclastogenesis. Arthritis Rheum 54: 158-168, 2006.

36. De Benedetti F, Rucci N, Del Fattore A, et al. Impaired skeletal development in interleukin-6-transgenic mice: a model for the impact of chronic inflammation on the growing skeletal system. Arthritis Rheum 54: 3551-3563, 2006.

37. Liu XH, Kirschenbaum A, Yao S, Levine AC. Cross-talk between the interleukin- 6 and prostaglandin $\mathrm{E}(2)$ signaling systems results in enhancement of osteoclastogenesis through effects on the osteoprotegerin/receptor activator of nuclear factor-\{kappa\}B (RANK) ligand/RANK system. Endocrinology 146: 1991-1998, 2005. 
38. Zuwała-Jagiełło J, Pazgan-Simon M, Simon K, Warwas M. Advanced oxidation protein products and inflammatory markers in liver cirrhosis: a comparison between alcohol-related and HCVrelated cirrhosis. Acta Biochim Pol 58: 59-65, 2011.

39. Li WY, Jiang YF, Jin QL, Zhang H, Feng XW, Niu JQ. Immunologic characterization of posthepatitis cirrhosis caused by $\mathrm{HBV}$ and HCV infection. J Biomed Biotechnol 2010: 138237, 2010.

40. Wiest R, Weigert $J$, Wanninger $J$, et al. Impaired hepatic removal of interleukin-6 in patients with liver cirrhosis. Cytokine 53: 178183, 2011.

(C) 2011 The Japanese Society of Internal Medicine http://www.naika.or.jp/imindex.html 